\title{
Comparison of brain transcriptome profiles of short-lived and long-lived species of Nothobranchius
}

\author{
Zulfiia Guvatova \\ Center for Precision Genome Editing \\ and Genetic Technologies for \\ Biomedicine \\ Engelhardt Institute of Molecular \\ Biology, Russian Academy of Sciences \\ Moscow, Russia \\ guvatova.zulfiya@mail.ru \\ Alexander Frolov \\ Laboratory for Ecological Monitoring \\ of Nuclear Power Plant (NPP) Regions \\ and Bioindication \\ Russia A.N. Severtsov Institute of \\ Ecology and Evolution \\ Moscow, Russia
}

\author{
George Krasnov \\ Center for Precision Genome Editing \\ and Genetic Technologies for \\ Biomedicine \\ Engelhardt Institute of Molecular \\ Biology, Russian Academy of Sciences \\ Moscow, Russia \\ gskrasnov@mail.ru \\ Nataliya Gladysh \\ Veterinary biology faculty \\ MSAVM\&B - MVA named after \\ K.I. Skryabin \\ Moscow, Russia \\ natalyagladish@gmail.com
}

\author{
Sergey Simanovsky \\ Laboratory for Ecological Monitoring \\ of Nuclear Power Plant (NPP) Regions \\ and Bioindication \\ Russia A.N. Severtsov Institute of \\ Ecology and Evolution \\ Moscow, Russia \\ sergey.a.simanovsky@gmail.com \\ Anna Kudryavtseva and Genetic Technologies for Biomedicine \\ Center for Precision Genome Editing \\ Engelhardt Institute of Molecular \\ Biology, Russian Academy of Sciences \\ Moscow, Russia \\ rhizamoeba@mail.ru
}

\begin{abstract}
Annual fishes of the genus Nothobranchius from East Africa is a promising vertebrate model in comparative and ageing studies. Nothobranchius show accelerated growth and age-related changes at all levels of organization. The present work aims to compare brain transcriptome profiles of shortlived $N$. rachovii with longer-lived $N$. korthausae and $N$. guntheri.
\end{abstract}

\section{Keywords - Nothobranchius, RNA-seq, brain aging}

\section{Introduction}

In recent years, annual fishes of the genus Nothobranchius have become powerful vertebrate animal models for the study of aging. Due to the short lifespan, Nothobranchius manifest changes associated with age such as skin color fade, spine curvature, weight loss, decline in cognitive abilities and an increased incidence of tumours in a few months after hatching. Moreover, killifish are sensitive to variations in water temperature, which allows easy manipulating their rate of aging under laboratory conditions.

Another important aspect is that different species of the genus Nothobranchius show differences in life expectancy, making them useful for comparative ageing research [1]. It has been suggested that the lifespan determination in the genus Nothobranchius depends on the rainfall pattern in the biotope of origin and differences in rearing and maintenance conditions. Maximum lifespan for these fish have been observed that range from 3 for $N$. furzeri to 28 months for $N$. guentheri.

It is known that there are conserved features linked to brain aging of mammals, such reduced adult neurogenesis and down-regulation of synaptic and axonal proteins expression. For well-known short-lived teleost fish $N$. furzeri brain aging has also been shown [2]. On the other hand, significant brain regenerative properties and adult neurogenesis were observed for other teleost fish as medaka and zebrafish [3]. The aim of the present study was to compare brain transcriptome profiles of several short- and longer-lived species of the genus Nothobranchius.

\section{Materials and Methods}

\section{Fish strains and maintenance}

N. rachovii Biera-98, N. korthausae MI TZN 08-4, and N. guntheri AS were obtained from commercial supplier (Peter Covar, Brno) and acclimated to the Center for Precision Genome Editing and Genetic Technologies for Biomedicine at the Engelhardt Institute of Molecular Biology. To avoid the effects of maintenance conditions, animals were bred under identical conditions. Fish were kept in 2 litre tanks at $27^{\circ} \mathrm{C}$ under a $14 \mathrm{~h}$ : $10 \mathrm{~h}$ day:night regime and fed twice a day with Chironomus larvae

RNA isolation, library preparation and transcriptome sequencing

Total RNA was isolated from brain of male and female specimens aged 3 months (adult) using MagNA Pure Compact RNA Isolation Kit on MagNA Pure Compact Instrument (Roche, Switzerland) according to manufacturer's protocol. RNA quantity and quality were evaluated using a Qubit 2.0 fluorometer (Life Technologies, USA) and Agilent 2100 Bioanalyzer (Agilent Technologies, USA). Samples passed quality (RIN $>8$ ) were used for subsequent experiments. Double stranded cDNA library was prepared by using TruSeq RNA Library Prep Kit v2 following manufacturer's protocol from $1 \mathrm{mg}$ of total RNA. Quality of prepared cDNA libraries were checked with bioanalyzer Agilent 2100 using High Sensitivity DNA chip (Agilent Technologies, USA). Then, libraries were normalized to $4 \mathrm{nM}$, pooled together in equal volumes, and sequenced on the NextSeq ${ }^{\text {TM}} 500$ system (Illumina, USA).

\section{NGS Data Processing}

About 100 million paired-end Illumina reads $(2 * 150 \mathrm{bp})$ were derived for each of 3 Nothobranchius species (N. rachovii, $N$. korthausae, N. guntheri). Reads were trimmed with trimmomatic 0.39 , and then reads were processed using cutadapt 2.8 to ensure the complete removal of adapters. Next, we performed transcriptome assembly using Trinity 2.9.1. For this purpose, we pooled libraries. The assembled transcripts were analyzed for bacterial contamination using Kraken 2 (MiniKraken DB_8GB), and bacterial contigs have 
been removed. Overall statistics have been calculated using QUAST. The completeness of the assembled transcriptomes was evaluated using BUSCO 4.0.5 (Vertebrata and Actinopterygii OrthoDB v10 datasets). The assembled transcripts were annotated using Trinotate 3.2.0 pipeline. Next, we evaluated gene/transcript expression profiles and calculated ExN50 statistics. For this purpose, reads were mapped to the assembled transcripts using bowtie2 2.4.1 and then transcript/gene expression levels were inferred by RSEM 1.3.3. The derived read counts were transformed into FPKM values.

Next, we tried to identify orthologous gene groups for all 3 organisms using OrthoFinder. For this purpose, we translated the derived transcripts using TransDecoder and selected the longest ORF for each gene. The derived amino acid sequences and selected transcript were supplied to OrthoFinder. To reduce CPU time, we limited this analysis only to annotated genes. Finally, for each orthologous gene group, we compared gene expression levels (in terms of FPKM) between 3 organisms. Because of inevitable presence of the experimental biases between organisms (sequencedependent efficacy of PCR amplification, sequencing), only 3 -fold or greater expression level changes were considered as significant.

\section{Results}

The overall lengths of the assemblies varied from 393 to $458 \mathrm{Mb}$; the total number of genes varied from 56 to 71 thousand. From 19 to 26 thousand genes were successfully annotated with Trinotate. About $4 \%$ transcripts were marked by Kraken as contaminants. According to the results of BUSCO analysis, the completeness of the assemblies varied from $91 \%$ to $94 \%$ (Vertebrata dataset) and from $82 \%$ to $86 \%$ (Actinopterygii dataset; this suggests that Vertebrata dataset is more appropriate for Nothobranchius sp.). Using OrthoFinder, we successfully found orthologous groups for 54\% annotated genes (all 3 species), 84\% (at least 2 species).

Differential expression (DE) analysis showed that genes associated with chromatin remodeling were the most differentially expressed. According to the results, the genes
$C B X 1 A, C B X 7 A$ were up-regulated (counts per million $(C P M)>3$, false discovery rate $(F D R)<0.05)$ in short-lived $N$. rachovii by 8 times compared with long-lived $N$. guntheri and 5 times compared to $N$. korthausae. We also observed the down-regulation of enhancer of zeste 1(EZH1) expression levels in $N$. rachovii $(C P M>3, F D R<0.05)$ when compared to $N$. korthausae, but not to $N$. guntheri. The change of expression of these genes with age was also observed in human brain [4]. Pathway enrichment analysis revealed a difference in expression of gene associated with «Ribosome», «RNA processing» and «Lysosome» GO terms. We also found that short-lived fish had reduced expression of genes related to such age-associated pathways as Wnt and Noth signaling pathways. Thus, the results obtained in the work might contribute to better understanding of differences in lifespan between species of this genus.

\section{ACKNOWLEDGMENT}

This work was supported by grant $075-15-2019-1660$ from the Ministry of Science and Higher Education of the Russian Federation.

\section{REFERENCES}

[1] T. Genade M. Benedetti, E. Terzibasi, P. Roncaglia, D.R. Valenzano, A. Cattaneo, A. Cellerino. "Annual fishes of the genus Nothobranchius as a model system for aging research.," Aging Cell, vol. 4, no. 5, pp. 223-33, Oct. 2005, doi: 10.1111/j.1474-9726.2005.00165.x.

[2] M. Baumgart M. Groth, S. Priebe, A. Savino, G. Testa, A. Dix, R. Ripa, F. Spallotta, C. Gaetano, M. Ori, E. T. Tozzini, R. Guthke, M. Platzer, and A. Cellerino. "RNA-seq of the aging brain in the short-lived fish $\mathrm{N}$. furzeri - conserved pathways and novel genes associated with neurogenesis.," Aging Cell, vol. 13, no. 6, pp. 965-74, Dec. 2014, doi: 10.1111/acel.12257.

[3] P. M. Loerch, T. Lu, K.A. Dakin, J.M. Vann, A. Isaacs, C. Geula, J. Wang, Y. Pan, D.H. Gabuzda, C. Li, T.A. Prolla, B.A. Yankner. "Evolution of the aging brain transcriptome and synaptic regulation.," PLoS One, vol. 3, no. 10, p. e3329, Oct. 2008, doi: 10.1371/journal.pone.0003329.

[4] S. Horvath Y. Zhang, P. Langfelder, R.S. Kahn, M.P. Boks, K. van Eijk, L.H. van den Berg, R.A. Ophoff. "Aging effects on DNA methylation modules in human brain and blood tissue.," Genome Biol., vol. 13, no. 10, p. R97, Oct. 2012, doi: 10.1186/gb-2012-13-10-r97. 